\title{
A Prediction Model of Structural Settlement Based on EMD-SVR-WNN
}

\author{
Xianglong Luo $\mathbb{D}$, , Wenjuan Gan $\mathbb{D}^{1},{ }^{1}$ Lixin Wang $\mathbb{D}^{2},{ }^{2}$ Yonghong Chen $\mathbb{D},{ }^{1}$ and Xue Meng $\mathbb{i D}^{1}$ \\ ${ }^{1}$ School of Information Engineering, Chang'an University, Xi'an 710064, China \\ ${ }^{2}$ China Railway First Survey and Design Institute Group Co., Ltd., Xi'an 710043, China
}

Correspondence should be addressed to Wenjuan Gan; wjgan@chd.edu.cn and Lixin Wang; 458601714@qq.com

Received 19 August 2020; Revised 24 November 2020; Accepted 5 December 2020; Published 22 December 2020

Academic Editor: Xinyu Ye

Copyright ( 92020 Xianglong Luo et al. This is an open access article distributed under the Creative Commons Attribution License, which permits unrestricted use, distribution, and reproduction in any medium, provided the original work is properly cited.

Timely and accurate prediction of structural settlement is of great significance to eliminate the hidden danger of structural and prevent structural safety accidents. Since the deformation monitoring data usually is nonstationary and nonlinear, the deformation prediction is a difficult problem in the structural monitoring research. Aiming at the problems in the structural deformation prediction model and considering the internal characteristics of deformation monitoring data and the influence of different components in the data on the prediction accuracy, a combined prediction model based on the Empirical Mode Decomposition, Support Vector Regression, and Wavelet Neural Network (EMD-SVR-WNN) is proposed. EMD model is used to decompose the structure settlement monitoring data, and the settlement data can be effectively divided into relatively stable trend terms and residual components of random fluctuation by energy matrix. According to the different characteristics of random items and trend items, WNN and SVR methods are, respectively, used for prediction, and the final settlement prediction is obtained by integrating the prediction results. The measured ground settlement data of foundation pit in subway construction is used to test the performance of the model, and the test results show that the prediction accuracy of the combined prediction model proposed in this paper reaches $99.19 \%$, which is $77.30 \%$ higher than the traditional SVR, WNN, and DBN-SVR models. The experimental results show that the proposed prediction model is an effective model of structural settlement.

\section{Introduction}

With the rapid development of information technology, automatic monitoring of structural deformation has become an important way to ensure structural safety [1-3]. Based on the monitoring data, the prediction of structural deformation to prevent potential safety hazards during construction and operation of large buildings (such as subway, tunnel, and bridge) has become a research hotspot of structural deformation monitoring in recent years [4]. As the scale of the project increases, higher requirements are put forward on the construction and operation safety of the structure. The processing of the real-time monitoring in the field of structural deformation monitoring also becomes especially important. According to the accurate and effective analysis of the real-time structural monitoring data, the structural deformation prediction model can be effectively established, which is of great significance to ensure the structural safety.

At present, the prediction models in the field of structural deformation prediction can be roughly divided into single prediction models and combined prediction models. The single prediction methods such as regression analysis method, time series analysis [5], grey system theory [6], and artificial intelligence method [7-10] are commonly used in the structural deformation prediction [11]. Li et al. [12] applied the GM $(1,1)$ grey system theory model to the prediction of subgrade deformation and explained the application conditions of the theory with engineering examples. However, the grey system requires the data sequence to have nonnegative characteristics, and the data accumulation has the rule of grey index, which limits the wide application of Grey Theory in structural deformation prediction. Zhenget al. [13] proposed the applicability principle of 
predicting the deformation of highway high slope based on stepwise regression analysis and verified the proposed method is an effective prediction method. Time series analysis requires the data to be linear and stable, and the deformation monitoring data in practical engineering are complex and nonlinear, which will affect the prediction accuracy. Chen and Wei [14] introduced fuzzy time series into deformation analysis and prediction. Experimental results show that fuzzy time series can be effectively applied to deformation prediction, and the prediction accuracy is improved. Liu and Hao [15] used the wavelet neural network to establish the prediction model. The result shows that this method has the great nonlinear function approaching capability. However, there are some shortcomings in the applicability and generalization of the single model. In view of the problems of a single model, some improved models $[16,17]$ and combined models have achieved better results.

In order to further improve the prediction accuracy of structural deformation monitoring data, researchers have proposed a hybrid model that combines two or more prediction models, which can correspondingly optimize the processing of nonstationary time series signals. Liu et al. [18] proposed a Least-Square Support Vector Machine (LS-SVM) model based on the phase space reconstruction for forecasting nonlinear time series of dam deformation, and the experimental results indicate that the forecasting performance of the proposed method is significantly superior to that of the traditional multiple regression method. Jingzhou et al. [19] established the Kalman-ARIMA-GARCH (Generalized Autoregressive Conditional Heteroskedasticity) model to predict bridge structural deformation, and the results showed that the modified model can effectively improve the prediction accuracy. Su et al. [20] established a deformation prediction model by combining support vector machine, phase space reconstruction, wavelet analysis, and Particle Swarm Optimization (PSO). The results show that the method has high modeling efficiency and prediction accuracy. Jiang et al. [21] proposed the Least-Squares-Support Vector Machine-Markov Chain (LS-SVM-MC) model, which was based on the Markov Chain predicted by Least-Square Support Vector Machine (LS-SVM) to correct the error, and applied the model to the deformation analysis of an arch dam. The results show that the model has high prediction accuracy. Zhao et al. [22] proposed an enhanced empirical wavelet transform (MSCEWT) based on the maximum-minimum length curve method. This method is used to decompose the signal into a series of intrinsic mode functions (IMFs). Cao et al. [23] proposed a combined prediction model for dam deformation prediction. The model uses the EMD method to decompose the original signal and then uses the correlation vector machine and ARIMA model to predict the decomposed components, respectively. Ren et al. [24] used the EMD method to decompose the original deformation monitoring data into subcomponents with different characteristics and then used GA algorithm to optimize the parameters of the WNN model to predict the values of different components, and finally fuse the predicted values of each subsequence. In summary, when predicting deformation monitoring data, decomposing the signal into internal components with different characteristics is of great significance to the improvement of prediction accuracy.

Aiming at the poor applicability of a single model and the characteristics of deformation data, this paper proposes a combined prediction model based on Empirical Mode Decomposition, Support Vector Regression, and Wavelet Neural Network (EMD-SVR-WNN). Firstly, the EMD model is used to decompose data into different components with different physical characteristics. EMD decomposition can effectively decompose monitoring data into trend and random fluctuation term according to energy distribution. Secondly, the Support Vector Regression (SVR) prediction model [25] can overcome the shortcomings caused by highdimensional model construction in dealing with nonlinear problems. Thirdly, the Wavelet Neural Network (WNN) prediction model $[26,27]$ is used to predict the trend term. The WNN prediction model combines the advantages of wavelet transform and neural network model, and it combines good time-frequency localization characteristic and self-learning functions of neural network theory, which makes it have the best ability of function approximation and fault tolerance and overcome the shortcomings of slow learning speed and low network training success rate in traditional neural networks. Finally, the prediction results of the SVR model and WNN model are fused to obtain the final model prediction results. The results show that the EMDSVR-WNN model proposed in this paper can achieve good results and has high prediction accuracy, and it is an effective structural deformation prediction model, which can provide important decision support for structural safety prevention.

The rest of this paper is organized as follows. Section 2 reviews the related work in the prediction of structural settlement and gives a brief introduction to the related methodology. The EMD-SVR-WNN prediction model is introduced in Section 3. Section 4 discusses the prediction results and performance analysis. Section 5 makes the conclusion of this paper and gives some recommendations.

\section{Methodology}

2.1. Empirical Mode Decomposition. Empirical Mode Decomposition (EMD) is an adaptive signal decomposition method, which can adaptively decompose nonlinear and nonstationary time series into trend and random terms, including several Intrinsic Mode functions (IMF) with the same characteristic scale and a residual component. IMF must satisfy two conditions at the same time when decomposing [28-30]. Firstly, the total number of extreme points is equal to or at most one different point from the total number of zero crossing points in all time periods of decomposing signals. Secondly, in any time period, the mean value of the upper envelope composed of local maximum points and the lower envelope composed of local minimum points is 0 . For any structural deformation sequence, the specific decomposition procedure is as follows:

(1) Find the extreme point of the original signal $x(n)$, and all the extracted local maximum and minimum points were interpolated by cubic spline 
interpolation to obtain the upper $\left(U_{\max }(n)\right)$ and lower $\left(V_{\min }(n)\right)$ envelopes.

(2) Calculate the mean value of the upper and lower envelope, and equation (1) is obtained:

$$
m(n)=\frac{U_{\max }(n)+V_{\min }(n)}{2} .
$$

(3) Calculate the difference between the signal and the mean value of the envelope, and equation (2) is obtained:

$$
T(n)=x(n)-m(n) .
$$

(4) Determine whether $T(n)$ satisfies the above two IMF conditions. If the condition is met, take $T(n)$ as the first component of $x(n)$, i.e., $c_{1}$. If the above condition is not met, $T(n)$ will be taken as the new original signal to continue the iteration until the above conditions are met.

(5) Calculate the value of the residual component, it can be noted as follows:

$$
r_{1}(n)=x(n)-c_{1} .
$$

(6) Take the residual component $r_{1}(n)$ as the original signal and repeat steps (1) to (5). Then, continuously decompose the 1,2..., $m$ IMF components that satisfy the condition, and the iteration stops until the residual component is a monotonic function. It can be noted as follows:

$$
x(n)=\sum_{i=1}^{m} c_{i}+r_{m}(n) .
$$

2.2. Support Vector Regression. Support Vector Machine (SVM) is a pattern recognition method based on statistical theory [31-33], which was first used to solve the classification problem in pattern recognition. Support Vector Regression (SVR) is the application of support vector in the field of function regression. SVR is a typical nonlinear forward feed network with hidden units, which can realize regression prediction of time series. The structure settlement data is time series data with random, nonstationary, and complex characteristics. The SVR model can overcome the defects caused by the construction of high-dimensional model. And it also has a solid statistical theory, and fewer data samples can be used to train data [34]. Therefore, the SVR model is widely used to deal with nonlinear and nonstationary time series regression prediction problems.

The main idea of support vector regression is to map data to a high-dimensional feature space through nonlinear mapping and then conduct linear regression on the data in this high-dimensional space [35]. Given a set of data points $\left(x_{1}, y_{1}\right),\left(x_{2}, y_{2}\right), \ldots,\left(x_{m}, y_{m}\right)$ for prediction model, where $x_{i} \in R^{n}, y_{i} \in R^{n}$, and $m$ is the total number of the sampling points. A linear regression function can be noted as follows:

$$
f(x)=\omega^{T} \Phi\left(X_{i}\right)+b, \quad \omega \in R^{n}, b \in R,
$$

where $\omega$ is weight vector and $b$ is bias value; the $\omega$ and $b$ can be obtained by minimizing the following function:

$$
\begin{aligned}
& \min \left[\frac{1}{2}\|\omega\|^{2}+C \sum_{i=1}^{m} \xi_{i}+\xi_{i}^{*}\right], \\
& \text { s.t. }\left\{\begin{array}{l}
\left(\omega x_{i}+b\right)-y_{i} \leq \varepsilon+\xi_{i} \\
y_{i}-\left(\omega x_{i}+b\right) \leq \varepsilon+\xi_{i} \\
\xi_{i}, \xi_{i}^{*} \geq 0, \varepsilon \geq 0,
\end{array}\right.
\end{aligned}
$$

where $\|\omega\|^{2}$ is the expression that controls the function capacity, $C$ is the penalty coefficient, $\varepsilon$ is the insensitive loss function [33], and $\xi_{i}, \xi_{i}^{*}$ are, respectively, the upper and lower training error subject to the $\varepsilon$. The optimization problem of the above can be expressed as the following nonlinear regression function:

$$
\begin{aligned}
& f(x)=\sum_{i=1}^{l}\left(\alpha_{i}-\beta_{i}\right) K\left(x_{i}, x\right)+b, \\
& \text { s.t. } 0 \leq \alpha_{i} \leq C, 0 \leq \beta_{i} \leq C,
\end{aligned}
$$

where $\alpha_{i}$ and $\beta_{i}$ are Lagrange operators and $K\left(x_{i}, x\right)=\left(\Phi\left(x_{i}\right), \Phi(x)\right)$ is kernel function. The kernel functions commonly used at present include polynomial kernel function, radial basis kernel function, and sigmoid kernel function.

Different kernel functions have different advantages; choosing the appropriate kernel function is very important for constructing nonlinear regression prediction models. In the field of machine learning, the radial basis kernel function can effectively realize the nonlinear mapping from input space to high-dimensional feature space. The convergence domain of the radial basis kernel function is wide and the performance is stable, and it is suitable for arbitrary distributed samples. Therefore, combined with the characteristics of structural settlement deformation data, the radial basis kernel function is selected as the kernel function of the support vector regression model in this paper.

2.3. Wavelet Neural Network. Wavelet neural network (WNN) is based on the topology of BP neural network, the Wavelet basis function is selected for the transmission signals of hidden layer nodes in the Neural Network. WNN combines wavelet transform with neural network, and the advantages of them are fully utilized. The structure diagram of the wavelet neural network model is shown in Figure 1, where $x_{n}$ is the input parameter of the wavelet neural network, $y_{m}$ is the output parameter of the wavelet neural network, and $g_{i}(x)$ is the wavelet basis function. When the signal $h(x)$ is input in the wavelet neural network, the wavelet basis function $g$ is used for fitting, and equation (8) is obtained:

$$
\widehat{h}(x)=\sum_{n=1}^{N} w_{n} g\left(\frac{x-b_{n}}{a_{n}}\right),
$$




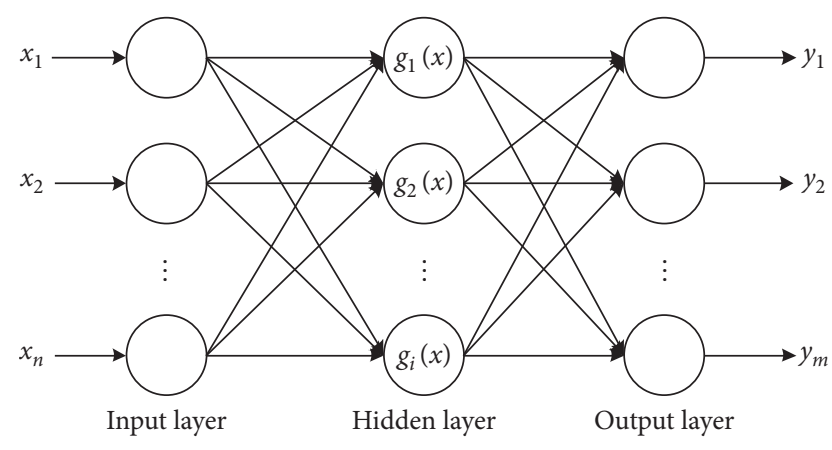

FIGURE 1: Wavelet neural network model structure.

where $\widehat{h}(x)$ is the fitted function, $N$ is the number of wavelet bases, $w_{n}$ is the weight of input layer and hidden layer, $a_{n}$ is the scale factor of the wavelet basis function, and $b_{n}$ is the translation factor of wavelet basis function.

The prediction process of the wavelet neural network model can be divided into two parts: training process and prediction process. The training process of the network is to find the parameters that make the network converge. The prediction process is to use the parameters obtained by network training to obtain the predicted value of the whole network. The training process of wavelet neural network is similar to the neural network method, which combines the forward propagation of signals with the backpropagation of errors. Wavelet neural network has strong learning ability and function approximation ability, and it overcomes the shortcomings of traditional neural network such as slow learning speed and low success rate of network training $[36,37]$. In view of these characteristics of wavelet neural network, this paper selects the wavelet neural network model to predict the trend term of monitoring data.

\section{Structural Settlement Deformation Prediction Model}

3.1. EMD-SVR-WNN Prediction Model. The EMD-SVRWNN prediction model proposed in this paper is based on empirical mode decomposition, support vector regression, and wavelet neural network model. The EMD decomposition model decomposes structural deformation data into different components with different physical characteristics and reduces the nonstationary characteristic of deformation monitoring data to a large extent. Then, different prediction models are constructed according to the internal characteristics of different components. SVR prediction model overcomes the shortcomings of neural network model, such as overfitting and easy to fall into local optimum. It is considered as an alternative method of artificial neural network [38] and has great advantages in dealing with nonstationary and nonlinear time series. Therefore, the SVR model is selected to predict the random term of the decomposed monitoring data. WNN prediction model combines the advantages of wavelet transform and BP neural network and has strong self-learning ability and localization of wavelet transform [39]. Therefore, the WNN model is adopted to predict the trend term of decomposed monitoring data.

In actual projects, monitoring data are generally obtained by automatic monitoring equipment. It is assumed that the original deformation monitoring data can be expressed as a two-dimensional matrix $X$, where $\mathrm{m}$ is the number of sensors and $\mathrm{n}$ is the number of sampling points for each sensor; then, the original data matrix can be expressed as $X \in R^{n \times m}$. Suppose the $k$ th column of the original data is selected for analysis, which is recorded as $X_{k}=\left[x_{1 k}, x_{2 k}, x_{3 k} \ldots, x_{n k}\right]$. Then, it is decomposed by EMD:

$$
X_{k}=\sum_{i=1}^{p} \mathrm{IMF}_{i}+r_{n}
$$

where $p$ represents the number of Intrinsic Mode Functions (IMF) of different feature scales and $r_{n}$ represents the residual component. In the process of signal decomposition, the frequency and energy distribution of the original signal will change. In order to further determine the trend term and random term of the signal, analyze the energy changes of different IMF components and extract the features of each different IMF component. The specific energy characteristic matrix solution steps are as follows:

(1) Firstly, the energy of $p$ IMF components is calculated according to

$$
E_{i}=\sum_{-\infty}^{\infty}|\mathrm{IMF}|_{i}^{2}, \quad(i=1,2, \ldots, p)
$$

(2) Then, the energy of each IMF component is normalized according to

$$
E=\sum_{i=1}^{p} E_{i}
$$

(3) The energy characteristic matrix can be expressed as follows:

$$
y=\left[\frac{E_{1}}{E}, \frac{E_{2}}{E}, \ldots, \frac{E_{i}}{E}\right] .
$$

Assuming that the energy feature matrix values of the IMF components of the front $f$ layer are less than the energy threshold $\varepsilon$, the IMF components of the front $f$ layer are used as the random term $X_{r}$, and the remaining IMF components are used as the trend term $X_{t}$ :

$$
\begin{aligned}
& X_{r}=\sum_{i=1}^{f} \mathrm{IMF}_{i}, \\
& X_{t}=\sum_{i=f+1}^{p} \mathrm{IMF}_{i} .
\end{aligned}
$$


SVR and WNN models are used to predict the random term $X_{r}$ and trend term $X_{t}$, respectively. The final model prediction result can be obtained by fusing the prediction result $\widehat{X}_{r}$ of the random term and the prediction result $\widehat{X}_{t}$ of the trend term:

$$
\widehat{S}=\widehat{X}_{r}+\widehat{X}_{t} .
$$

If $d_{m}$ is the target output value, the mapping relationship between target input $\bar{X}$ and target output $Y$ is $R^{z} \longrightarrow R$, and the data matrix $X$ and $\bar{Y}$ is as follows:

$$
\begin{aligned}
& \hat{X}=\left[\begin{array}{ccccc}
d_{r 1} & d_{r 2} & d_{r 3} & \cdots & d_{r z} \\
d_{r 2} & d_{r 3} & d_{r 4} & \cdots & d_{r, z+1} \\
d_{r 3} & d_{r 4} & d_{r 5} & \cdots & d_{r, z+2} \\
\vdots & \vdots & \vdots & \ddots & \vdots \\
d_{r, n-z} & d_{r, n-z-1} & d_{r, n-z-2} & \cdots & d_{r n}
\end{array}\right], \\
& \hat{Y}=\left[\begin{array}{c}
d_{z+1} \\
d_{z+2} \\
d_{z+3} \\
\vdots \\
d_{n+1}
\end{array}\right] .
\end{aligned}
$$

In formula (15), nis the length of the data sequence and $z$ is the embedding dimension.

The specific steps and flow charts of the EMD-SVR-WNN prediction model proposed in this paper are shown in Figure 2. The implementation process of the method is as follows:

Step1: by performing EMD decomposition on the structural settlement monitoring data, low-frequency components and high-frequency components are obtained. The value of the energy characteristic matrix is used to determine whether the IMF component belongs to trend $X_{t}$ or random data $X_{r}$, according to formulas (10)-(13).

Step2: the random term $X_{r}$ uses the SVR prediction model to predict; select the first $z$ data samples in the sample as the training set to predict $n-z$ data, and the random term prediction result is recorded as $\hat{X}_{r}$.

Step 3: the trend term $X_{t}$ uses the WNN prediction model to predict; select the first $z$ data samples in the sample as the training set to predict $n-z$ data, and the trend term prediction result is recorded as $\widehat{X}_{t}$.

Step 4: the prediction results of random and trend term are fused to obtain the final prediction results according to formula (14).

3.2. Performance Evaluation Criteria. The evaluation criteria to measure the error of the prediction results included root mean square error (RMSE), mean absolute error (MAE), mean square percentage error (MSPE), and mean absolute percentage error (MAPE). The RMSE and MAPE are selected as evaluation criteria of EMD-SVR-WNN model in this paper. The formulas are as follows:

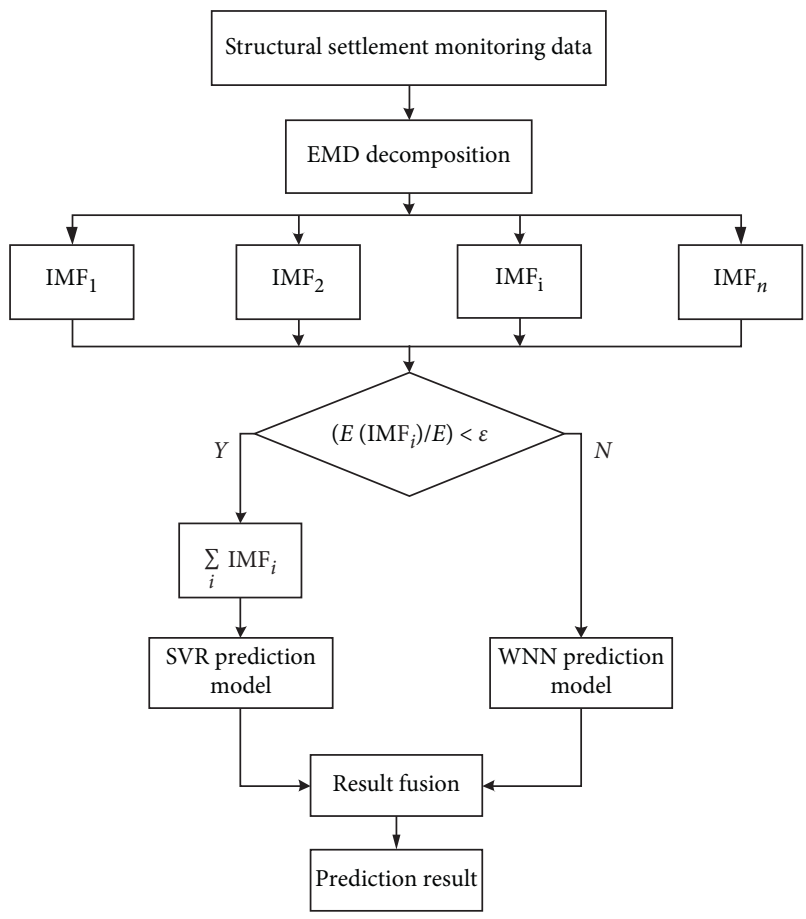

FIGURE 2: EMD-SVR-WNN prediction model flowchart.

$$
\begin{aligned}
& \text { RMSE }=\sqrt{\frac{1}{N} \sum_{i=1}^{N}\left(y_{i}-\hat{y}_{i}\right)^{2},} \\
& \text { MAPE }=\frac{1}{N} \sum_{i=1}^{N}\left|\frac{y_{i}-\hat{y}_{i}}{y_{i}}\right| \times 100 \% .
\end{aligned}
$$

where $y_{i}$ is the real value of the structural deformation, $\hat{y}_{i}$ is the predicted value of the structural deformation, and $N$ is the number of predicted samples.

\section{Prediction Results and Performance Analysis}

To evaluate the effectiveness of the proposed model, the experiment was carried out using the ground settlement data of a subway station in TianJin, and the settlement data contained 1000 sampling points. It can be seen from Figure 3 that the monitoring data reflects the actual characteristics of cumulative vertical displacement of the surface. In this paper, the cumulative variation of surface settlement of 800 sampling points in a section of a construction subway is selected for experimental testing. The first 500 data are selected as training samples to predict the last 300 data.

4.1. Analysis of EMD-SVR-WNN Model Results. In order to illustrate the necessity of EMD decomposition, the amplitude-frequency characteristics of the settlement monitoring data to be tested are analyzed firstly, and the Fast Fourier Transform method is used to transform the time domain into the frequency domain. It can be seen from Figure 4 that the settlement monitoring data is mainly concentrated in the low-frequency part. This phenomenon shows that the data 


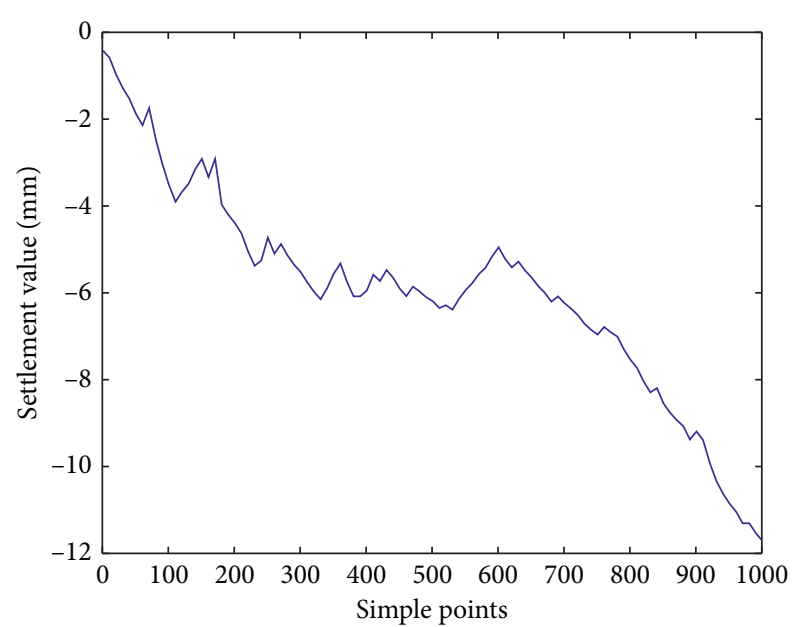

Figure 3: Original settlement data.

fluctuation caused by external factors or the characteristic information of the settlement data itself contains different frequencies.

In order to further analyze the characteristics of the settlement monitoring data, the power spectral density of the data is analyzed, as shown in Figure 5. Through the analysis of Figure 5, this shows that the set of settlement data can be decomposed into multiple components with different frequencies. At the same time, because the set of settlement data changes slowly and is nonlinear and unstable, selecting a suitable decomposition method can reduce the instability of the signal and extract the characteristic information of the signals at different frequencies. Data prediction based on the abovementioned signal decomposition can greatly improve the prediction accuracy.

EMD decomposition method is suitable for processing nonlinear and nonstationary signals. The settlement signals are decomposed into high-frequency components and lowfrequency components by EMD decomposition, and then different components are predicted by appropriate prediction methods, so as to reduce the impact of the instability of settlement data on the prediction results. The trend and random terms of data are obtained by EMD decomposition. The decomposition results are shown in Figure 6.

It can be seen from Figure 6 that the EMD decomposition divides the data into 5 layers, of which the first few layers show obvious high-frequency characteristics, and the higher the level of the data, the lower the frequency. At the same time, the data after EMD decomposition becomes more stable and smooth, and the data is adaptively divided into trend items and random items. This paper extracts the energy features of the abovementioned 5-layer IMF components and normalizes the energy values of different IMF components to construct an energy feature vector matrix. The constructed energy feature vector is shown in Table 1 .

It can be seen from the energy characteristic values in Table 1 that the energy value of the 5th layer IMF component accounts for the largest proportion and can be regarded as the main energy of the signal. From the perspective of signal decomposition, the trend term, that is, the low-frequency

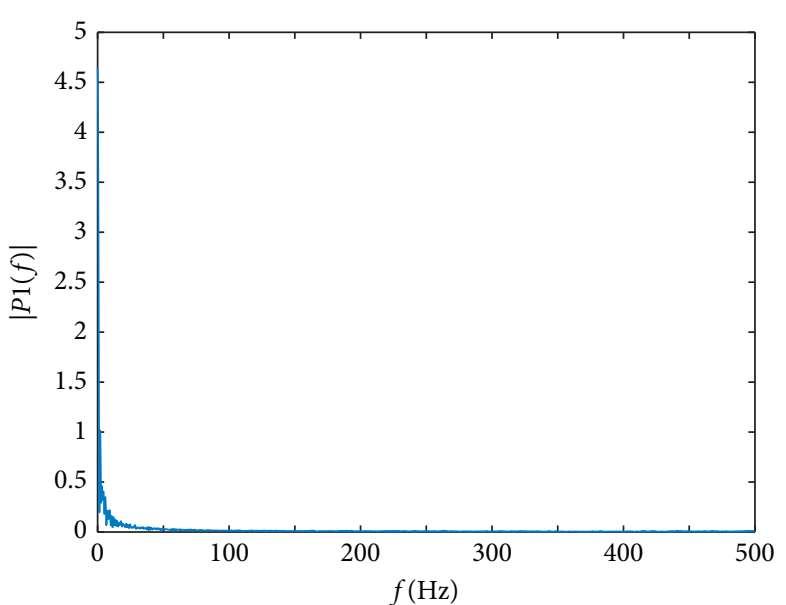

Figure 4: Amplitude-frequency characteristic analysis.

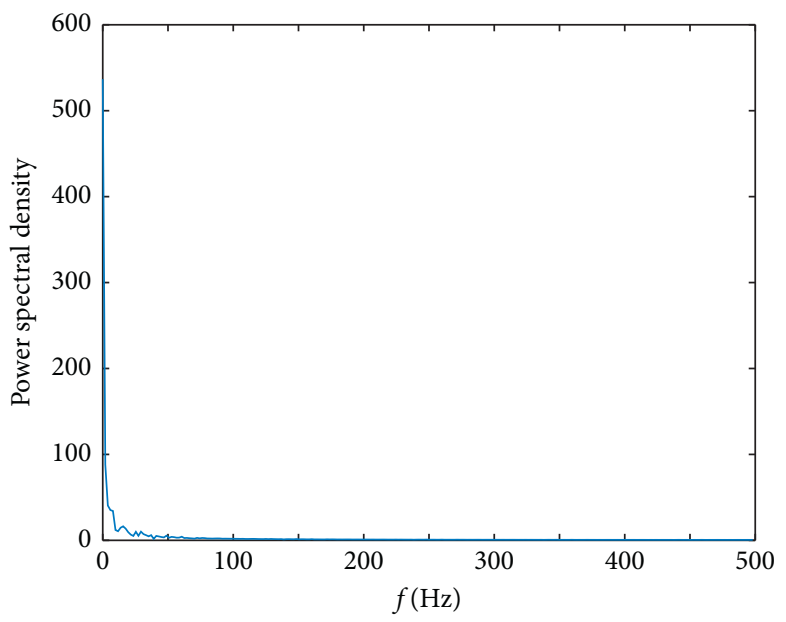

Figure 5: Power spectral density analysis.

component contains the main energy of the signal, and the high-frequency component accounts for the secondary energy of the signal. Therefore, in this article, the IMF5 component is used as the low-frequency component, and the remaining IMF components are superimposed as the highfrequency component of the data sequence. The result is shown in Figures 7 and 8.

After the EMD decomposition of the deformation monitoring data, the data is divided into four IMF components and one residual IMF5. The components after EMD decomposition show more obvious physical characteristics. The analysis and prediction of settlement deformation data can achieve better results on the basis of the EMD decomposition. In this paper, the first four IMF components are firstly predicted by the SVR model. After superimposing the first four layers components, the first 500 data are selected as training samples, while the last 300 data are selected as test samples, and the predicted results are compared with the real values. The predicted results of SVR model are shown in Figure 9.

It can be seen from Figure 9 that the predicted value of the SVR model is very close to the real value of the 

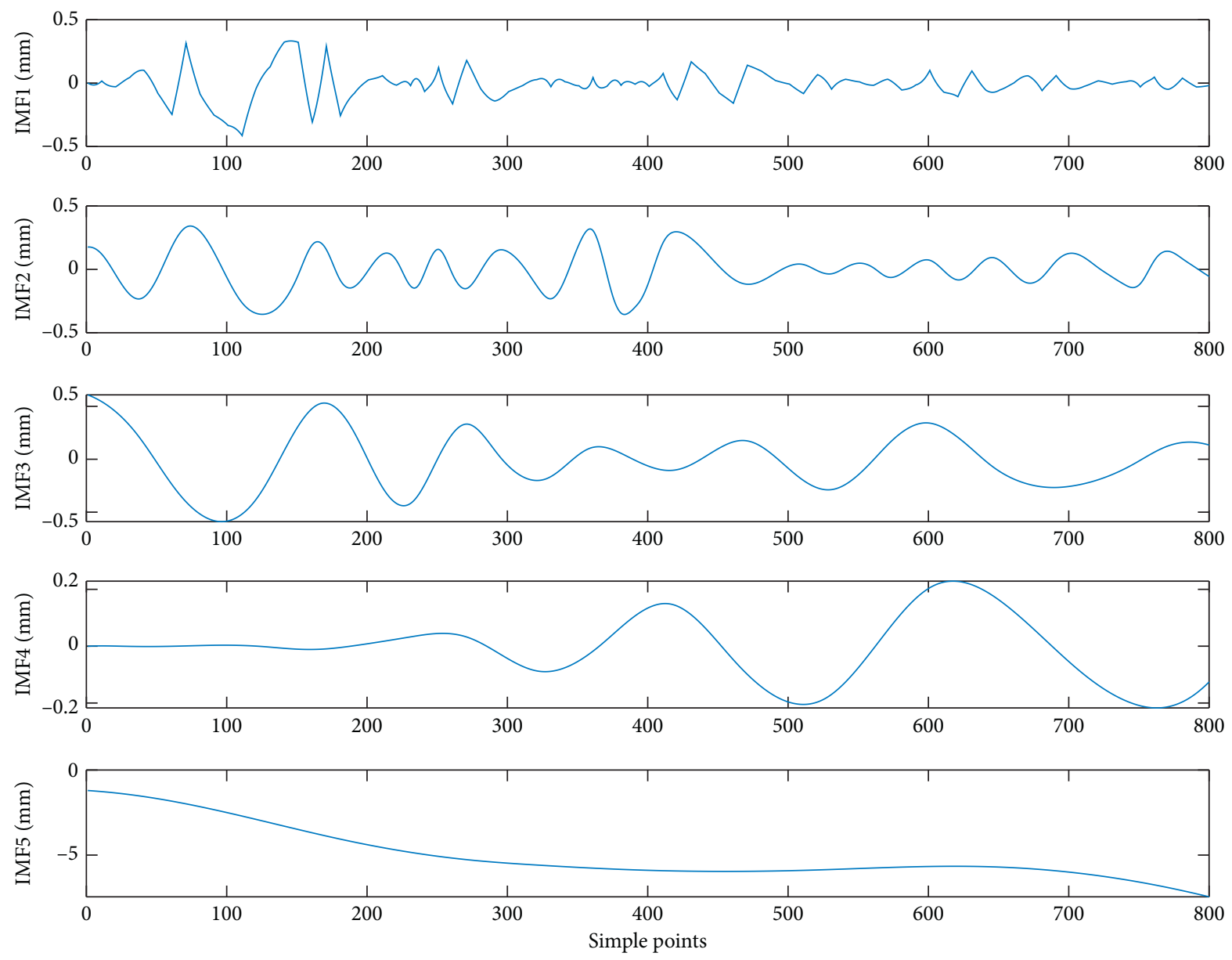

FIGURE 6: EMD decomposition result.

TABLE 1: Signal energy characteristic value of each layer.

\begin{tabular}{lccccc}
\hline$E_{i} / E$ & $E_{1} / E$ & $E_{2} / E$ & $E_{3} / E$ & $E_{4} / E$ & $E_{5} / E$ \\
\hline Energy & 0.0699 & 0.1736 & 0.0269 & 0.1406 & 0.5890 \\
\hline
\end{tabular}

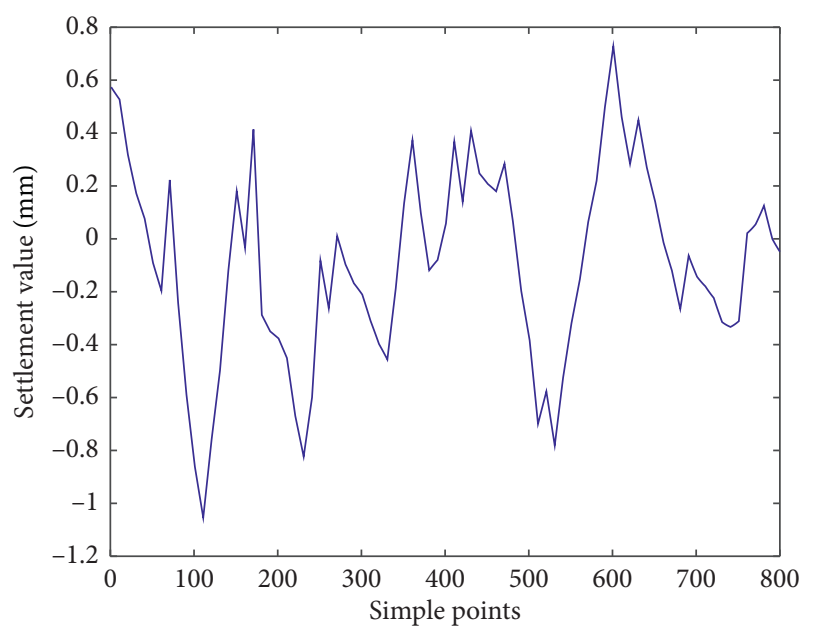

Figure 7: The first four layers' component. monitoring data, and only the prediction results at the extreme points have a certain deviation. The maximum absolute error between the predicted value and the real value is $0.3090 \mathrm{~mm}$, and the minimum absolute error between the predicted value and real value is $0.00007 \mathrm{~mm}$. The result shows that the trend of the predicted deformation value is basically consistent with the real value. After the prediction of the random term, the trend term is predicted by the WNN model. The first 500 data of the fifth layer component are selected as training samples to predict the last 300 data. The predicted results of the WNN model are shown in Figure 10.

It can be seen from Figure 10 that the predicted value of the WNN model is almost identical with the real monitoring value, and the prediction accuracy is high. The maximum absolute error between the predicted value and the real value is $0.0898 \mathrm{~mm}$, and the minimum absolute error between the predicted value and the real value is $0.00003 \mathrm{~mm}$.

Finally, the prediction results of SVR and WNN are fused to obtain the final prediction results of the EMD-SVR-WNN model. The final prediction results are shown in Figure 11.

It can be seen from Figure 11 that the prediction results of the proposed model are very close to real values, and the prediction accuracy is very high. It can be concluded from the experimental results that the RMSE of the proposed 


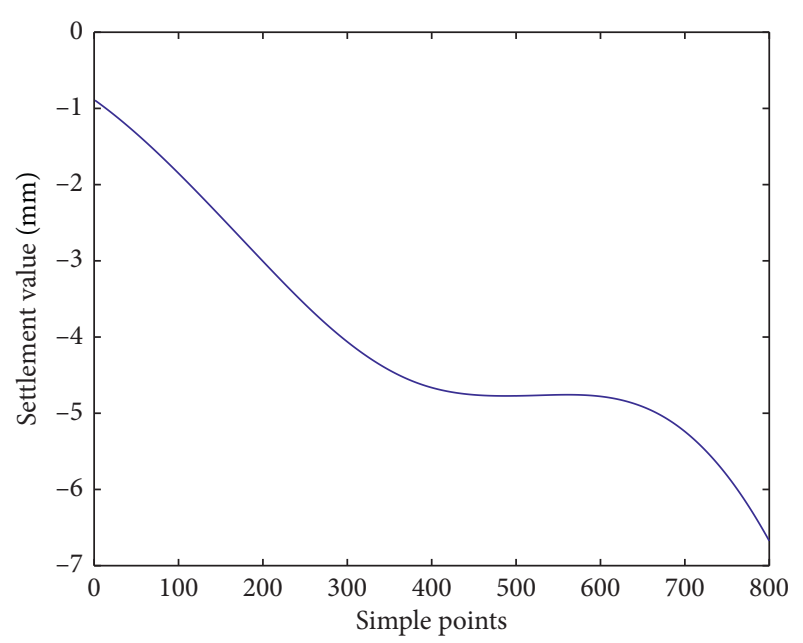

Figure 8: The fifth layer component.

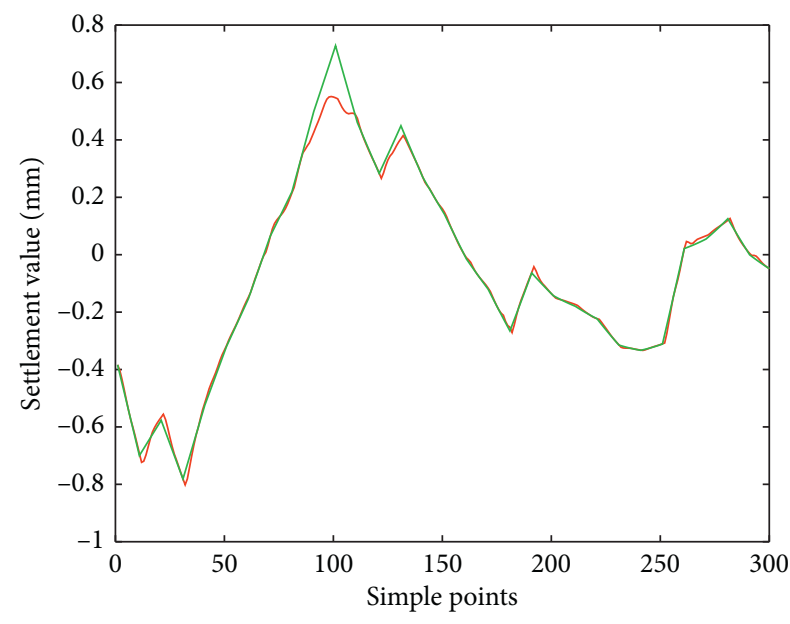

- Real value

Figure 9: Random term prediction result.

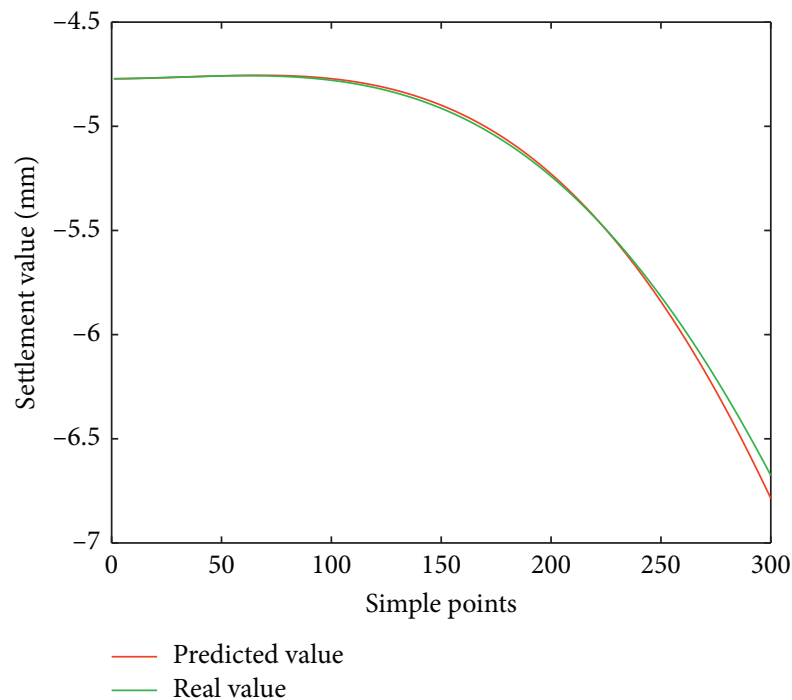

Figure 10: Trend term prediction result.

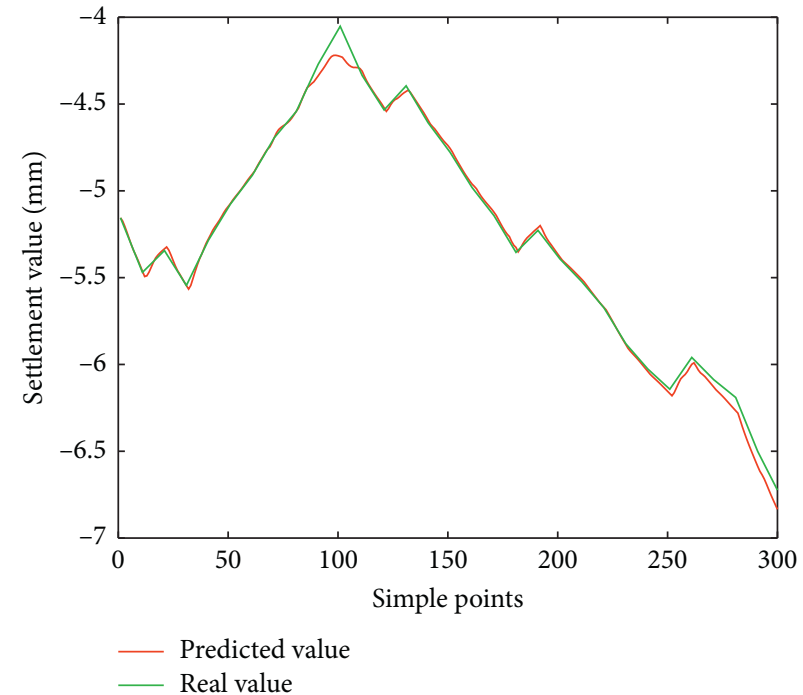

FIgURE 11: EMD-SVR-WNN prediction result.

model is $0.0531 \mathrm{~mm}$ and the precision reaches $99.19 \%$, which indicates that the proposed model is an effective settlement prediction model. The result also indicates that the EMDSVR-WNN model can be well applied to the field of structural deformation prediction and achieve higher prediction accuracy.

4.2. Performance Analysis. In order to evaluate the effectiveness of our proposed approach, the SVR, WNN, and DBN-SVR models are selected to compare with the EMDSVR-WNN model proposed in this paper. In the SVR model, the nuclear function is configured as " $r b f$, " the number of iterations is 10,000 , and the penalty factor is taken as 0.01 . In the WNN model, the number of iterations is 1000 , the number of the hidden layer nodes is 3. In the DBN-SVR model, the number of network layers in the DBN model is set as 3, the number of iterations is 200 , the kernel function of the SVR classifier is " $r b f$," the number of iterations is 10,000 and the penalty factor is 0.01 . The computer configuration for the experiment is shown Table 2 .

Under the experimental environment and the parameter conditions in this paper, the performance comparison of different prediction models is shown in Table 3 and the comparison of different prediction models with real values is shown in Figure 12.

It can be seen from Figure 12 that the SVR model has the worst prediction performance; the DBN-SVR and WNN model are better than the SVR model, while they show weakness compared with the EMD-SVR-WNN model. The prediction value of the proposed model is almost coincided with the monitoring data. The experimental results show that the proposed EMD-SVR-WNN prediction model has better prediction performance, and it is an effective model for structural deformation prediction.

It can be seen from Table 3 that the prediction accuracy of the EMD-SVR-WNN model, respectively, improves $87.10 \%, 81.46 \%$, and $63.35 \%$ compared with the SVR, WNN, 
TABLE 2: Experimental environment.

\begin{tabular}{lc}
\hline CPU & Intel(R) core (TM) i5-6200U @2.30 GHZ \\
\hline RAM & $4 \mathrm{~GB}$ \\
Operating system & Window (64) \\
Matlab & R2018a \\
\hline
\end{tabular}

Table 3: Performance comparison of different prediction models.

\begin{tabular}{lccc}
\hline Model & MAPE & RMSE & Running time $(\mathrm{s})$ \\
\hline SVR & 0.0628 & 0.5669 & 68.2459 \\
WNN & 0.0437 & 0.2730 & 7.2856 \\
DBN-SVR & 0.0221 & 0.3946 & 192.6416 \\
EMD-SVR-WNN & 0.0081 & 0.0531 & 73.3579 \\
\hline
\end{tabular}

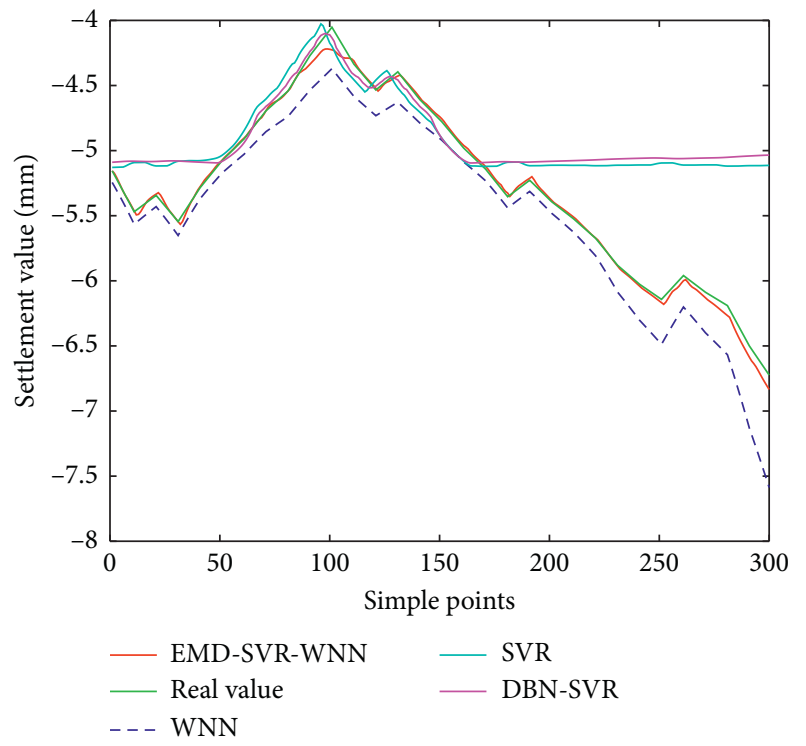

Figure 12: Comparison of different prediction models.

and DBN-SVR models. The average prediction accuracy is $77.30 \%$ higher than other prediction models. Computation complexity is also an important criterion for model evaluation. It usually refers to the resources required at runtime after being written into an executable program. The higher the complexity of the model, the longer the running time; running time is used to discuss computation complexity in this paper. DBN-SVR and WNN models have the longest and shortest running time, respectively, but the accuracy is low. The proposed model has a relatively short running time while ensuring prediction accuracy. The experimental results show that the proposed EMD-SVR-WNN prediction model has higher prediction accuracy than other prediction models, and it is an effective structural deformation prediction model.

\section{Conclusions and Recommendations}

Structural deformation is inevitable during the construction process, so it is of great significance to avoid accidents through structural deformation prediction. Most of the existing structural deformation prediction models lack the mining of internal features within the data, and there is a lack of discussion on the applicability of the prediction model. Aiming at the above problems, this paper proposes a combined prediction model based on Empirical Mode Decomposition, Support Vector Regression, and Wavelet Neural Network (EMD-SVR-WNN). Based on the analysis of the measured data at Tianjin Station, the following conclusions can be drawn:

(1) The data is decomposed into different components with different physical characteristics through the EMD decomposition method. According to the different characteristics of different signal components, the appropriate prediction model is selected, which can effectively improve the accuracy of deformation prediction.

(2) According to the characteristics of random items and trend items, the SVR model has great advantages in dealing with nonlinear problem and can overcome the defects caused by high-dimensional model construction. The WNN prediction model combines the advantages of wavelet transform and neural network model. SVR and WNN are finally selected for prediction, respectively.

(3) Finally, the prediction results of the SVR model and WNN model are fused to obtain the final model prediction results. Combined with practical engineering, the results show that the EMD-SVR-WNN model proposed in this paper can achieve good results and has high prediction accuracy; this is of great significance to the study of structural deformation in engineering.

\section{Data Availability}

The data used to support the findings of this study are available from the corresponding author upon request.

\section{Conflicts of Interest}

The authors declare that there are no conflicts of interest regarding the publication of this paper.

\section{Acknowledgments}

This research was partly supported by the National Key R\&D Program of China (2018YFC0808706).

\section{References}

[1] J. Xin, J. Zhou, S. Yang, X. Li, and Y. Wang, "Bridge structure deformation prediction based on GNSS data using kalmanARIMA-GARCH model," Sensors, vol. 18, no. 1, p. 298, 2018.

[2] G. Su, Z. Yan, and G. Chen, "Forecasting deformation time series of surrounding rock for tunnel using Gaussian process," in Proceedings of the International Conference on Computational Intelligence \& Software Engineering, Wuhan, China, December 2010.

[3] G. Chen, Y. L. Ma, H. Sun et al., "Research on the method of deformation monitoring prediction based on fuzzy neural 
netwrok," in Proceedings of the Electrical And Control Engineering (ICECE), IEEE, Wuhan, China, January 2010.

[4] V. A. Kontogianni and S. C. Stiros, "Predictions and observations of convergence in shallow tunnels: case histories in Greece," Engineering Geology, vol. 63, no. 3-4, pp. 333-345, 2002.

[5] H. Zhang, X. Shi, and L. F. Lai, "Research on time series analysis based deformation prediction model," Advanced Materials Research, vol. 250-253, pp. 2888-2891, 2011.

[6] D. L. Qiao and M. Zhao, "Deformation prediction based on time series analysis and grey system theory," Advanced Materials Research, vol. 368-373, pp. 2147-2152, 2011.

[7] H. Chen, Z. Zeng, and H. Tang, "Landslide deformation prediction based on recurrent neural network," Neural Processing Letters, vol. 41, no. 2, pp. 169-178, 2015.

[8] F. Kang, J. Liu, J. Li et al., "Concrete dam deformation prediction model for health monitoring based on extreme learning machine," Structural Control \& Health Monitoring, vol. 24, pp. 1-11, 2017.

[9] X. Qu, J. Yang, and M. Chang, "A deep learning model for concrete dam deformation prediction based on RS-LSTM," Journal of Sensors, vol. 2019, no. 1, pp. 1-14, Article ID 4581672, 2019.

[10] J. Lai, J. Qiu, Z. Feng et al., "Prediction of soil deformation in tunnelling using artificial neural networks," Computational Intelligence \& Neuroscience, vol. 8, p. 33, 2016.

[11] G. Tan, H. Liu, Y. Cheng, B. Liu, and Y. Zhang, "Prediction method for the deformation of deep foundation pit based on neural network algorithm optimized by particle swarm," in Proceedings of the International Conference on Transportation, IEEE, Katowice-Ustron, Poland, June 2012.

[12] L. Hui, Y. Echuan, Y. Min et al., "Research on the limitations of deformation prediction with GM $(1,1)$ model," in Proceedings of the International Workshop on Intelligent Systems \& Applications IEEE, Loughborough, UK., July 2009.

[13] W. Zheng, D. Xiaodong, W. Ganlin, and Z. Lei, "The applicability study of predicting highway slope deformation based on stepwise regression analysis method," Modern Transportation Technology, vol. 14, no. 4, pp. 10-17, 2017.

[14] W. Chen and C. Wei, "Deformation analysis and prediction based on fuzzy time series," Applied Mechanics and Materials, vol. 405-408, pp. 2448-2451, 2013.

[15] P. P. Liu and Y. J. Hao, "The research of tailings pond deformation displacement prediction based on wavelet neural network," Applied Mechanics and Materials, vol. 271-272, pp. 1726-1730, 2012.

[16] W. Deng, H. Liu, J. Xu et al., "An improved quantum-inspired differential evolution algorithm for deep belief network," IEEE Transactions on Instrumentation and Measurement, vol. 69, no. 7, 2020.

[17] W. Deng, J. Xu, H. Zhao, Y. Song et al., "A novel gate resource allocation method using improved PSO-based QEA," IEEE Ransactions on Intelligent Transportion Systems, vol. 52, 2020.

[18] H. Z. Liu, S. L. Wang, and J. Y. Liu, "LS-SVM prediction model based on phase space reconstruction for dam deformation," Advanced Materials Research, vol. 663, pp. 55-59, 2013.

[19] X. Jingzhou, Z. Jianting, Y. Simon et al., "Bridge structure deformation prediction based on GNSS data using KalmanARIMA-GARCH model," Sensors, vol. 18, Article ID s18010298, 2018.

[20] H. Su, X. Li, B. Yang, and Z. Wen, "Wavelet support vector machine-based prediction model of dam deformation,"
Mechanical Systems and Signal Processing, vol. 110, pp. 412427, 2018.

[21] Y. Z. Jiang, H. Z. Liu, and J. Y. Liu, "LS-SVM-Markov model for dam deformation prediction," Applied Mechanics and Materials, vol. 423-426, pp. 1144-1149, 2013.

[22] H. Zhao, S. Zuo, M. Hou et al., "A novel adaptive signal processing method based on enhanced empirical wavelet transform technology," Sensors, vol. 18, Article ID s18103323, 2018.

[23] E. H. Cao, T. F. Bao, and Y. T. Liu, "EMD-RVM-Arima based prediction model of dam deformation and its application," Water Resources and Hydropower Engineering, vol. 49, pp. 59-64, 2018.

[24] C. Ren, Y. J. Liang, and G. F. Pang, "The empirical mode decomposition and genetic algorithm-wavelet neural network for slope deformation prediction research," Journal of Geomatics Science and Technology, vol. 31, pp. 551-555, 2014.

[25] X. S. Liu and L. Yu, "Application of improved SVR in prediction of side slope displacement of the dam," Bulletin of Surveying and Mapping, vol. 6, pp. 122-125, 2018.

[26] Y. M. Pan, Q. Z. Zhang, and P. Q. Xue, "The method and application of time series prediction based wavelet neural network, advanced Materials Research," Journal of Harbin Engineering University, vol. 328-330, pp. 2312-2317, 2011.

[27] C. Yao and Y. Yu, "An improved wavelet neural network method for wind speed forecasting," Journal of Computational and Theoretical Nanoscience, vol. 10, no. 12, pp. 2860-2865, 2013.

[28] N. E. Huang, S. Zheng, S. R. Long et al., "The empirical mode decomposition and the Hilbert spectrum for nonlinear and non-stationary time series analysis," in Proceedings Mathematical Physical \& Engineering Sciences, vol. 454, pp. 903-995, 1971.

[29] B. Marxim Rahula Bharathi and A. R. Mohanty, "Time delay estimation in reverberant and low SNR environment by EMD based maximum likelihood method," Measurement, vol. 137, pp. 655-663, 2019.

[30] E. H. Cao, T. F. Bao, Y. T. Liu et al., "EMD-RVM-Arima based prediction model of dam deformation and its application," Water Resources and Hydropower Engineering, vol. 49, no. 12, pp. 59-64, 2018.

[31] C. Cortes and V. Vapnik, "Support-vector networks," $M a-$ chine Learning, vol. 20, no. 3, pp. 273-297, 1995.

[32] V. N. Vapnik, "An overview of statistical learning theory," IEEE Transactions on Neural Networks, vol. 10, no. 5, pp. 988-999, 1999.

[33] V. N. Vapnik, The Nature of Statistical Learning Theory, Springer, New York, NY, USA, 1995.

[34] O. Chapelle, V. Vapnik, O. Bousquet, and S. Mukherjee, "Choosing multiple parameters for support vector machines," Machine Learning, vol. 46, no. 1, pp. 131-159, 2002.

[35] S. Miao, Q. Zhu, L. Zhang et al., "A knowledge-guided landslide deformation prediction approach based on SVR," in Proceedings of the International Conference on Geoinformatics, IEEE, Fairfax VA, USA, August 2017.

[36] G. R. Pan and G. Chuan, "Wavelet neural network prediction method of deformation monitoring data," Journal of Geodesy and Geodynamics, vol. 27, no. 4, pp. 47-50, 2007.

[37] D. X. Niu and M. Xing, "A study on wavelet neural network prediction model of time series," Systems Engineering-Theory \& Practice, vol. 5, pp. 89-92, 1999.

[38] L. Zhou, H. Y. Wen, and Y. K. Han, "Application of grey prediction and Kalman filter in building settlement 
deformation analysis," Science of Surveying \& Mapping, vol. 39, 2014.

[39] C. Guan, P. B. Luh, L. D. Michel, Y. Wang, and P. B. Friedland, "Very short-term load forecasting: wavelet neural networks with data pre-filtering," IEEE Transactions on Power Systems, vol. 28, no. 1, pp. 30-41, 2013. 